\title{
Short communication: Factors affecting hair cortisol concentrations in lactating dairy cows
}

\author{
Tracy A. Burnett, ${ }^{*}$ Augusto M. L. Madureira, ${ }^{*}$ Bruna F. Silper, ${ }^{*}$ Audrey Nadalin, $\dagger$ Abdolmansour Tahmasbi, $\ddagger$ \\ Douglas M. Veira, $†$ and Ronaldo L. A. Cerri*1 \\ ${ }^{*}$ Faculty of Land and Food Systems, University of British Columbia, Vancouver, BC, V6T 1 Z4 Canada \\ †Agriculture \& Agri-Food Canada, Agassiz, BC, V0M 1A0 Canada \\ $\ddagger$ Faculty of Agriculture, Ferdowsi University of Mashhad, Mashhad, Iran 91775-1163
}

\section{ABSTRACT}

Cortisol has long been used as a marker of the stress response in animals. Cortisol can be analyzed from different media, most notably from the blood, saliva, and feces; however, the collection of cortisol from some of these media requires invasive procedures or excessive handling of the animals. Furthermore, it is not possible to capture long-term increases in circulating concentrations of cortisol from the blood, saliva, or feces. Hair cortisol has been found to be a reliable alternative for measuring chronic stress. With this emerging measure, appropriate sampling methodology must be developed and validated. The aim of this study was to determine the effects of hair color, sampling location, and processing method on cortisol concentrations in hair from lactating black and white Holstein cows $(\mathrm{n}=18)$. Furthermore, we aimed to measure the hair growth rates at different body locations $(\mathrm{n}=12)$ and test hair cortisol levels when resampled over short intervals $(\mathrm{n}=37)$. Both black- and white-colored hair was collected from the shoulder, top line, hip, and tail switch of Holsteins; due to breed characteristics only white hair was harvested from the tail switch. All samples were cleaned with water and isopropanol, and then ground in a ball mill or finely cut with scissors once dry. Cortisol was extracted with methanol before being measured using a commercially available ELISA kit. Concentrations of cortisol were greater in white than in black hair $(7.8$ \pm 1.1 vs. $3.8 \pm 1.1 \mathrm{pg} / \mathrm{mg}$ ). When only white samples were analyzed, hair from the tail switch had more cortisol than hair from the shoulder $(11.0 \pm 1.2$ vs. 6.2 $\pm 1.2 \mathrm{pg} / \mathrm{mg}$ ), whereas no difference was found when compared with the hip and top line. Samples ground with a ball mill had greater concentrations of cortisol extracted than those minced with scissors $(10.4 \pm 1.2$ vs. $4.7 \pm 1.2 \mathrm{pg} / \mathrm{mg}$ ). The growth rate of hair was sig-

Received June 4, 2014.

Accepted September 1, 2014.

${ }^{1}$ Corresponding author: ronaldo.cerri@ubc.ca nificantly greater at the tail switch compared with the hip and shoulder $(0.51 \pm 0.05$ vs. $0.04 \pm 0.05$ vs. 0.03 $\pm 0.05 \mathrm{~mm} / \mathrm{d}$ ). When hair was collected every $3 \mathrm{wk}$ after calving, a tendency was detected for multiparous cows to have greater concentrations of hair cortisol and significantly greater concentrations of cortisol on d 0 and 21 after calving compared with d 42, 84, and 126. In Holsteins, the hair on the tail switch is always white, grows more rapidly than other sites, and is sensitive enough to capture changes in cortisol over intervals as short as $3 \mathrm{wk}$, making it the ideal location for measuring hair cortisol.

Key words: hair cortisol, Holstein cow, stress

\section{Short Communication}

Cortisol is a key mediator of the stress response in animals, produced through the activation of the hypothalamic-pituitary-adrenal (HPA) axis during a stressful event. Activation of the HPA axis can cause many physiological changes during acute stress events (i.e., short-term or low-magnitude increases in cortisol) and promotes a shift of resources toward adaptive functions such as arousal, vigilance, and increased oxygenation and nutrition of the brain, heart, and skeletal muscles. As a consequence, it may downregulate nonadaptive functions such as eating, growth, and reproduction (Chrousos, 2009). Many common farm practices induce acute stress responses in dairy cattle, such as handling (Cook et al., 2000), transportation (Lay et al., 1996), regrouping (Friend et al., 1977), dehorning (Stafford and Mellor, 2005), and overstocking (Friend et al., 1979). Prolonged or excessive activation of the HPA axis may result in chronic stress that can cause additional behavioral (Chrousos and Gold, 1992) and somatic disorders (Chrousos et al., 1998; Charmandari et al., 2005). In dairy cattle, long-term increases in glucocorticoid concentrations have been linked to immunosuppression (Roth, 1985) and reduced fertility (Dobson and Esslemont, 2002). Comin et al. (2013) reported that cows that were clinically compromised (e.g., lami- 
nitis, metritis, or mastitis) had greater concentrations of cortisol than those that had an absence of disease.

Cortisol has been extensively used as a physiological measure of acute stress response in animals (Moberg and Mench 2000). Cortisol can be analyzed from many different media, most notably from the blood, saliva, and feces; however, handling and restraint of dairy cattle has also been shown to rapidly increase plasma cortisol, leading to confounding results (Cook et al., 2000). In addition, circulating cortisol follows a diurnal pattern in a pulsatile fashion, leading to substantial individual variation depending on the time of day (Thun et al., 1981). Although fecal samples are less responsive to small changes in circulating cortisol and reflect cortisol concentrations from 10 to $12 \mathrm{~h}$ prior to fecal collection, they still cannot be practically used to measure the long-term changes that are characteristic of chronic stress (Möstl et al., 2002).

Sampling concerns and the lack of a reliable method for measuring chronic stress in farm animals, has contributed to the need for noninvasive cortisol sampling methods that can reflect long-term increases in cortisol (Moberg and Mench, 2000). One promising sampling method is the use of hair to assess cortisol levels; this method has been used in rhesus macaques (Davenport et al., 2008), dairy cows (Comin et al., 2011), and dairy calves (González-de-la-Vara et al., 2011). Hair cortisol concentrations have been found to have a positive correlation with chronic stress found in humans in different adverse circumstances and uncertain health, such as hospitalized neonates (Yamada et al., 2007), chronic pain in adults (Van Uum et al., 2008), acute myocardial infarction in men (Pereg et al., 2011), and Cushing's syndrome (Thomson et al., 2010).

Bennett and Hayssen (2010) reported that cortisol concentrations differed depending on hair color in dogs, where black hair contained the least amount of cortisol compared with blonde- and agouti-colored hair. Some evidence has been reported that the location on the body the hair sample originates from may affect cortisol concentrations. Macbeth et al. (2010) found that hair collected from the neck of grizzly bears had significantly higher cortisol concentrations than hair collected from the shoulder, rump, and abdomen. In addition, research with beef cattle has shown that cortisol concentrations were highest when hair was collected from the tail switch (Moya et al., 2013). Furthermore, different methods have been used to process hair samples before analysis. The 2 main methods used for grinding include mincing the hair into small pieces using scissors (Koren et al., 2002; Accorsi et al., 2008) and the use of a ball mill to powder the hair (Moya et al., 2013).

The aim of our study was to determine the effect of hair color and sampling location on concentrations of cortisol in lactating Holstein dairy cows and to identify the most effective processing method for cortisol extraction from hair. The hypothesis was that white-colored hair would yield greater concentrations of cortisol and that a location effect would be evident. Furthermore, more finely processed hair, through the use of a ball mill, would have greater concentrations of cortisol extracted.

The current project was conducted at the University of British Columbia's Dairy Education and Research Centre in Agassiz, Canada, from March to October 2011 in experiment $1(\mathrm{n}=18)$, July 2011 to January 2012 in experiment $2(\mathrm{n}=12)$, and March 2011 to March $2012(\mathrm{n}=37)$ in experiment 3. The approximate average temperature and photoperiod during the entire experimental period were $10.5^{\circ} \mathrm{C}$ and $14.5 \mathrm{~h} / \mathrm{d}$ of light, $17.8^{\circ} \mathrm{C}$ and $14.5 \mathrm{~h} / \mathrm{d}$ of light, $7.4^{\circ} \mathrm{C} 9.5 \mathrm{~h} / \mathrm{d}$ of light, and $4.9^{\circ} \mathrm{C}$ and $9.5 \mathrm{~h} / \mathrm{d}$ of light for the spring, summer, fall, and winter seasons, respectively.

All procedures were carried out in accordance with the University of British Columbia's Animal Care Committee. In addition, animals used in this experiment were cared for as outlined by the guidelines provided by the Canadian Council of Animal Care.

All animals within the current study were housed indoors within the same freestall barn equipped with deep sand-bedded stalls. Animals were milked twice daily at 0500 and $1500 \mathrm{~h}$ and fed a TMR twice daily at approximately 0700 and $1600 \mathrm{~h}$. The TMR was formulated to meet or exceed the requirements of Holstein cows weighing $620 \mathrm{~kg}$, producing $45 \mathrm{~kg} / \mathrm{d}$ of $3.5 \% \mathrm{FCM}$ (NRC, 2001); the animals had ad libitum access to both TMR and water.

An initial set of animals consisted of 18 lactating Holstein dairy cows were used to test the effect of hair color, sampling location, and processing method on hair cortisol concentrations. The animals had an average of $3.2 \pm 1.9$ (mean $\pm \mathrm{SD}$ ) lactations (3 primiparous and 15 multiparous), producing $11,720 \pm 2,214 \mathrm{~kg}$ of milk, and an average of $163.0 \pm 123.9$ DIM. All 18 dairy cows were sampled at 4 different locations: shoulder, top line, hip, and tail switch (Figure 1); samples of both black and white hair were taken at each location, when available, with the exception of the tail switch because Holstein cattle only have white hair at this location. Hair samples collected from the body of the animal were carefully clipped with electric clippers (blade size 40, Oster, Rye, NY; 2-Speed Clipper, Andis, Sturtevant, WI). Hair from the tail switch was collected with scissors, due to its coarse nature, and the hair material closest ( $2.5 \mathrm{~cm}$ of hair) to the skin was kept for analysis. In both cases, the hair was cut as close to the skin as possible and stored at room temperature in the dark, in dry paper envelopes until processed. 


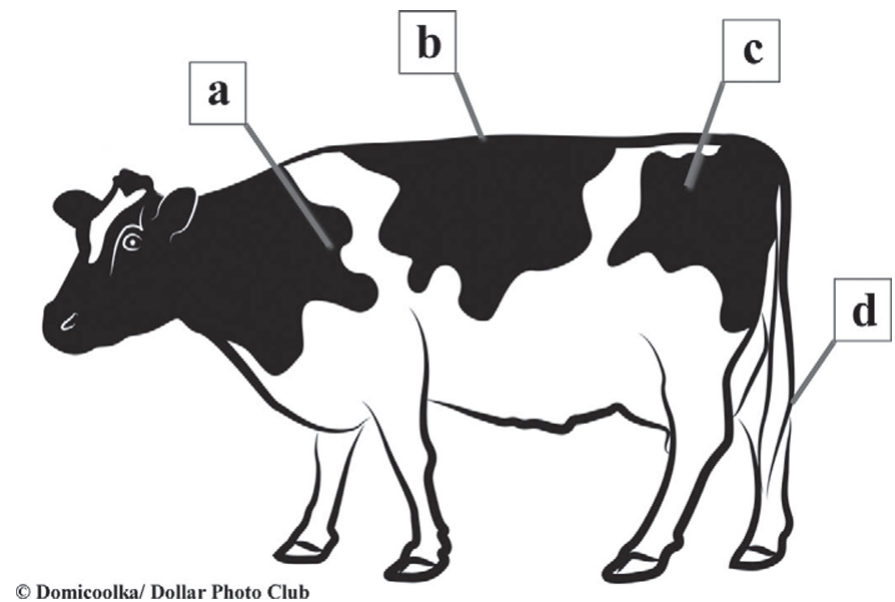

Figure 1. Hair sampling was done at the (a) shoulder, hair located at the scapula; (b) top line, hair located at the dorsal side of the vertebrae; (c) hip, hair located over the femur-ischium junction; and (d) tail switch, hair located at the distal end of the tail. Figure used with permission of Dollar Photo Club.

An additional 12 dairy cows were used to determine the rate at which hair grows in 3 different areas of the body: shoulder, hip, and tail switch. Only white hair was collected from each region. Hair from each area was cut as close as possible to the skin using electric clippers for the rump and shoulder or surgical scissors for the tail switch. Leftover hair stubble was dyed using black hair dye for a minimum of 30 min and washed off with cold water. Three weeks later, using a permanent red marker, hair was marked at the surface of the skin and plucked out using rat tooth tweezers. Hair was measured using a light table and a jeweler's eyepiece equipped with a small ruler; 10 to 12 hairs/location per cow were measured to the nearest $0.1 \mathrm{~mm}$. The amount of hair found between the dyed black hair and the red marker was defined as the hair growth length.

A third set of animals was collected to test for the effect of parity and DIM. Hair samples were collected from the tail switch of 37 lactating cows (18 primiparous and 19 multiparous) with no history of health disorders at 0, 21, 42, 84, and 126 DIM. All samples were processed using a ball mill following the previously described hair-collection protocol. The sequential collection of hair samples occurred every $3 \mathrm{wk}$ from calving until 126 DIM to test if samples could be properly collected and analyzed over short-term intervals from the same animal.

In preparation for analysis, samples were cleaned thoroughly of dirt, dander, and hairs of the wrong color. The samples were then washed with warm water for no longer than 3 min and let dry for a minimum of $1 \mathrm{~d}$ until completely dry. To prepare the hair samples used to test the effects of hair color, sampling location, and processing method, two 250-mg hair samples were weighed and washed in two 5 - $\mathrm{mL}$ washes of isopropanol of 3 min each, as suggested by Davenport et al. (2006). Both samples were left to dry for $5 \mathrm{~d}$ and one was finely cut (less than $3 \mathrm{~mm}$ ) using a pair of surgical scissors whereas the other was ground in a $10-\mathrm{mL}$ stainless steel milling cup with a $12-\mathrm{mm}$ stainless steel ball in a Retsch Mixer Mill MM400 ball mill (Retsch, Hannover, Germany) for $5 \mathrm{~min}$ at a frequency of 30 repetitions/s. Hair samples collected to test the effect of parity and DIM were prepared for analysis in the same manner as previously described; however, all samples were ground using the ball mill method only. Ground hair samples were then stored in the dark in glass jars at room temperature.

Extraction of hair cortisol was performed following the procedure of Koren et al. (2002), with modifications. We took $20 \pm 0.2 \mathrm{mg}$ of clean, dried, and ground bovine hair and weighed it into 7 -mL glass scintillation vials. Then, $1 \mathrm{~mL}$ of HPLC-grade methanol (EMD Chemicals, Darmstadt, Germany) was added. The vials were tightly capped, gently swirled to ensure the hair came into contact with the methanol, and sonicated for $30 \mathrm{~min}$. The samples were incubated overnight (New Brunswick Scientific Incubator Shaker, Edison, NJ) at $100 \mathrm{rpm}$ and $50^{\circ} \mathrm{C}$ to extract the steroids, and $0.8 \mathrm{~mL}$ of the original volume of methanol was pipetted into a 2-mL microcentrifuge tube and evaporated at $45^{\circ} \mathrm{C}$ under a stream of ultrapure nitrogen gas. The samples were reconstituted in $100 \mu \mathrm{L}$ of the PBS supplied with the assay kit.

Hair cortisol was analyzed using a commercially available assay kit designed for salivary cortisol (Salimetrics Expanded Range, High Sensitivity 1-E3002, State College, PA). The minimal concentration of cortisol that can be distinguished from the 0 standard using this kit is $0.003 \mu \mathrm{g} / \mathrm{dL}$. Samples were aliquoted into wells in duplicate $(25 \mu \mathrm{L})$, and absorbance measured using a wavelength of $450 \mathrm{~mm}$ in a microplate plate reader (Biorad xMark, Hercules, CA). The average inter- and intra-assay CV was 7.4 and $3.5 \%$, respectively.

Prior to statistical analyses, all data were checked for normality using probability distribution plots. All statistical analyses were performed using SAS (version 9.4; SAS Institute Inc., Cary, NC). To test for differences in cortisol concentrations for hair color, location of the body, and grinding method, as well as the analysis for sequential collections (parity and DIM), data were analyzed by ANOVA using the MIXED procedure. Differences between grinding methods only used samples of white hair. The hair growth measurements were pooled within animal and analyzed by ANOVA using the GLM procedure. 
Table 1. Effect of color, sampling location, and grinding method on concentrations of cortisol extracted from hair samples collected from black and white Holstein cows ${ }^{1}$

\begin{tabular}{lcc}
\hline & $\begin{array}{c}\text { Concentration } \\
\text { of cortisol } \\
\text { Item }\end{array}$ & $P$-value \\
\hline Color & $3.8 \pm 1.1$ & $<0.001$ \\
Black & $7.8 \pm 1.1$ & 0.36 \\
White & $4.8 \pm 1.1$ & 0.03 \\
Location (black and white hair) & $5.1 \pm 1.1$ & \\
Shoulder & $5.8 \pm 1.1$ & $<0.001$ \\
Top line & $6.2 \pm 1.2^{\mathrm{a}}$ & \\
Hip & $8.9 \pm 1.2^{\text {ab }}$ & $9.5 \pm 1.2^{\text {ab }}$ \\
Location (only white hair) & $11.0 \pm 1.2^{\mathrm{b}}$ & \\
Shoulder & $10.4 \pm 1.2$ & $4.7 \pm 1.2$ \\
Top line & & \\
Hip & & \\
Tail switch & & \\
Grinding method & & \\
Sull mill & & \\
a,b Values with different superscripts in the same column and within an item are significantly different $(P<$ \\
0.05).
\end{tabular}

The overall cortisol concentrations measured within our experiment $(5.7 \pm 1.7 \mathrm{pg} / \mathrm{mg})$ were within the range reported for lactating dairy cattle by Gonzálezde-la-Vara et al. (2011; $12.15 \pm 1.85 \mathrm{pg} / \mathrm{mg})$, but greater than those reported by Comin et al. (2011; 2.5 $\pm 0.10 \mathrm{pg} / \mathrm{mg}$ ) from dairy cattle and Moya et al. (2013; $2.35 \pm 0.176 \mathrm{pg} / \mathrm{mg}$ ) from beef cattle. Values obtained from the current study may be greater than previously found by Comin et al. (2011) due to the difference in hair-grinding method used. Furthermore, the values reported here were greatly different than those found in dairy heifer calves $(114.5 \pm 14.43 \mathrm{pg} / \mathrm{mg}$; González-dela-Vara et al., 2011) and male rhesus macaques (110.3 $\pm 10.2 \mathrm{pg} / \mathrm{mg}$; Davenport et al., 2006), suggesting that developmental stages and species greatly affect the amount of cortisol found in hair.

A difference in cortisol concentrations was found for hair of different colors $(P<0.001$; Table 1$)$. White hair had greater concentrations of cortisol than black hair. This difference was found irrespective of the location and the analysis excluded the data from the tail switch because of the lack of black hair. Similarly, Gonzálezde-la-Vara et al. (2011) also described black hair as having approximately half the concentration of cortisol as white hair from dairy cows. In addition, differences in hair cortisol affected by color have been reported in dogs, where yellow hair had greater concentrations of cortisol than agouti and black hair from German Shepherds (Bennett and Hayssen, 2010). Reasons for this difference are not well understood but could be related to mechanisms associated with melanocyte development (Slominski et al., 2004), differentiation (Roulin et al., 2008), or simply a question of physical space within the hair shaft, as white or yellow hair have less pigmentation. Hair color may have an effect on skin temperature, and therefore increase the blood flow through the microvasculature, compromising the dermal papilla and root sheaths of hair follicles; however, animals in our study were housed indoors, away from direct sunlight. Although it has been suggested that season may affect hair cortisol concentrations (Comin et al., 2011) by changing the rate of hair growth and the hair growth cycle (Courtois et al., 1996), the effects of photoperiod and temperature have not been shown in cattle.

Concentrations of cortisol from both colors did not differ among the shoulder, top line, and hip $(P=0.36$; Table 1). When only white was included in the analysis to include the tail switch, a difference between the tail switch and shoulder was found $(P=0.01)$, but not between the tail switch and the hip or top line (Table 1). These results for white hair are consistent with a recent study performed on beef cattle by Moya et al. (2013), where it was also reported that hair from the tail switch had greater concentrations of cortisol than the head and shoulder, whereas hair from the neck and hip had greater concentrations of cortisol compared with the shoulder. In our study, the hair from the top line and hip were not different than from the shoulder. The causes for the marked differences between the tail switch and shoulder are unclear; however, it has been suggested that the increased hair growth rate found at the tail switch may be the driving factor for increased cortisol concentrations (Moya et al., 2013). Furthermore, Moya et al. (2013) reported that hair from the tail switch had a stronger correlation to both salivary 
and fecal cortisol concentrations than hair from the head, neck, shoulder, and hip.

The processing method of the hair significantly affected the amount of cortisol extracted from each sample. Samples processed with a ball mill had greater cortisol concentrations extracted $(P<0.001)$ compared with surgical scissors (Table 1). The increase in cortisol extracted from hair is likely caused by the increased surface area created by grinding the hair into much smaller pieces. Grinding methods have not previously been directly compared for cortisol extraction from hair samples from dairy cows, but studies using surgical scissors have reported overall smaller concentrations of cortisol (Comin et al., 2011, 2013).

The rate of hair growth is affected by location. The growth rate of the hair from the tail switch is over 10 times faster $(P<0.001)$ than the hair growth of the hips and shoulder, whereas the hip and shoulder have similar growth rates $(0.51 \pm 0.05$ vs. $0.04 \pm 0.05$ vs. $0.03 \pm 0.05 \mathrm{~mm} / \mathrm{d}$; Figure 2). The hair growth results from the tail switch were similar to values reported by Fisher et al. (1985) and Schwertl et al. (2003) from beef cattle. Resampling intervals of biological significance in lactating dairy cows, such as during the transition period, can only be achieved with the growth rate found at the tail switch.

Parity only tended to affect concentrations of cortisol in hair, where multiparous cows had slightly greater concentrations than primiparous cows $(9.6 \pm 0.3$ vs. $8.9 \pm 0.3 ; P=0.10)$. Such a small difference is unlikely to indicate a clear parity effect, although it is a common belief that primiparous cows may suffer more from stress than multiparous cows, particularly during the transition period. Within this portion of the experiment, hair was only collected from cows with no sign of clinical disease throughout the sampling period. In a sister study (Burnett, T., A. Madureira, B. Silper,

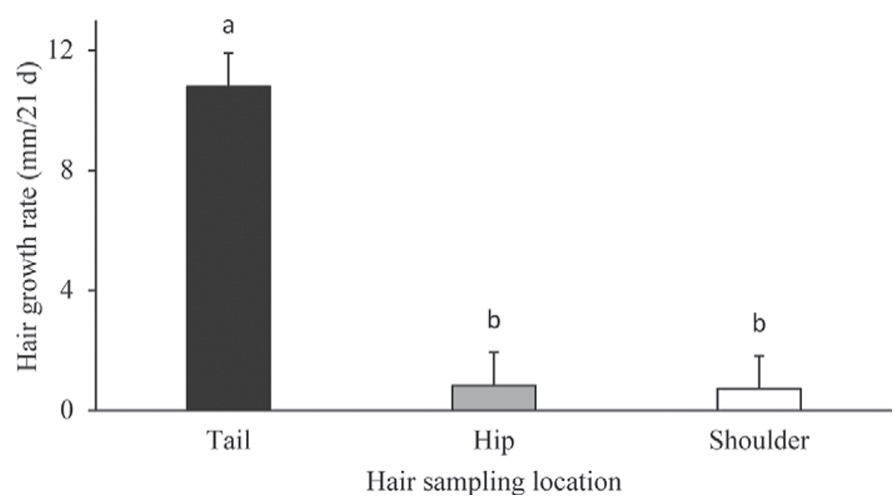

Figure 2. Hair growth rates at different body locations (mean \pm SE) collected from black and white Holstein cows. Different letters $(\mathrm{a}, \mathrm{b})$ indicate significant differences $(P<0.001)$.

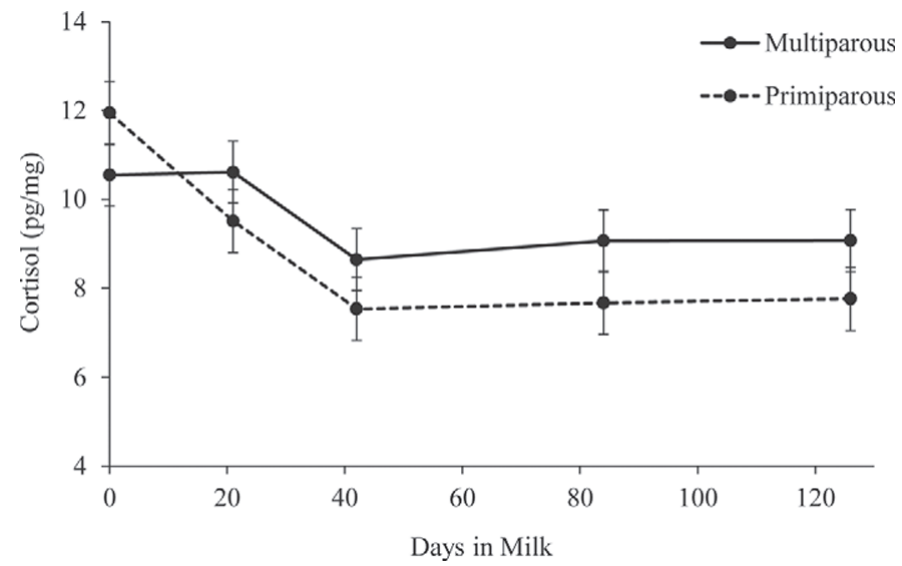

Figure 3. Effect of parity and DIM on concentrations of cortisol (mean \pm SE) extracted from hair samples collected from the tail switch of 37 lactating cows (18 primiparous and 19 multiparous) at $0,21,42,84$, and 126 DIM. A tendency for greater hair cortisol concentrations was noted in multiparous cows $(P=0.10)$. Days in milk affected concentrations of cortisol in hair $(P<0.001)$; d 0 and 21 had elevated concentrations of cortisol compared with the other collections. No interaction between parity and DIM was found $(P>0.16)$.

A. Nadalin, A. Tahmasbi, D. Veira, R. Cerri; unpublished data), it was observed that parity had a more pronounced effect, even when only including healthy animals. In addition, as DIM increased, a decrease in hair cortisol concentrations was observed $(P<0.001$; Figure 3), indicating that hair cortisol may be a valuable indicator of changes in stress during the weeks following parturition. No interaction between parity and DIM was found $(P>0.16)$.

The color of the hair, sampling location, and method of grinding largely affects the amount of cortisol extracted from hair. The current study suggests that the tail switch is the ideal location for hair sampling from black and white Holsteins for analysis of cortisol due to the consistent white-colored hair, greater growth rates, and easier access. In addition, a ball mill should be used to maintain consistent particle sizes among samples as well as to improve cortisol extraction. The creation of a more standardized method for hair sample collection for the analysis of cortisol in dairy cattle will improve the interpretation and consistency of results in different studies aiming to compare chronic stress in lactating dairy cows.

\section{ACKNOWLEDGMENTS}

This study was supported by a contribution from the Dairy Research Cluster Initiative (Dairy Farmers of Canada, Agriculture and Agri-Food Canada, the Canadian Dairy Network, and the Canadian Dairy Commission, Ontario, Canada). Partial funding was also provided by the Natural Sciences and Engineering 
Research Council (Ontario, Canada) grant NSERCRGPIN 418672-13. The authors also thank Nelson Dinn and all students that assisted with data collection at the University of British Columbia's Dairy Education and Research Centre.

\section{REFERENCES}

Accorsi, P. A., E. Carloni, P. Valsecchi, R. Viggiani, M. Gamberoni, C. Tamanini, and E. Seren. 2008. Cortisol determination in hair and faeces from domestic cats and dogs. Gen. Comp. Endocrinol. 155:398-402.

Bennett, A., and V. Hayssen. 2010. Measuring cortisol in hair and saliva from dogs: Coat color and pigment differences. Domest. Anim. Endocrinol. 39:171-180.

Charmandari, E., C. Tsigos, and G. P. Chrousos. 2005. Neuroendocrinology of stress. Annu. Rev. Physiol. 67:259-284.

Chrousos, G. P. 2009. Stress and disorders of the stress system. Nat. Rev. Endocrinol. 5:374-381.

Chrousos, G. P., and P. W. Gold. 1992. The concepts of stress and stress system disorders: overview of physical and behavioural homeostasis. JAMA 267:1244-1252.

Chrousos, G. P., D. Torpy, and P. W. Gold. 1998. Interactions between the hypothalamic-pituitary-adrenal axis and the female reproductive system: clinical implications. Ann. Intern. Med. 129:229-240.

Comin, A., T. Peric, M. Corazzin, M. C. Veronesi, T. Meloni, V Zufferli, G. Cornacchia, and A. Prandi. 2013. Hair cortisol as a marker of hypothalamic-pituitary-adrenal axis activation in Friesian dairy cows clinically or physiologically compromised. Livest. Sci. $152: 36-41$

Comin, A., A. Prandi, T. Peric, M. Corazzin, S. Dovier, and S. Bovolenta. 2011. Hair cortsiol levels in dairy cows from winter housing to summer highland grazing. Livest. Sci. 138:69-73.

Cook, C. J., D. J. Mellor, P. J. Harris, J. R. Ingram, and L. R. Matthews. 2000. Hands-on and hands-off measurement of stress. Pages 123-146 in The Biology of Animal Stress. G. P. Moberg and J. A. Mench, ed., CABI Publishing, Wallingford, UK.

Courtois, M., G. Loussouarn, S. Hourseau, and J. F. Grollier. 1996. Periodicity in the growth and shedding of hair. Br. J. Dermatol. 134:47-54.

Davenport, M. D., C. K. Lutz, S. Tiefenbacher, M. A. Novak, and J. S. Meyer. 2008. A Rhesus monkey model of self-injury: Effects of relocation stress on behavior and neuroendocrine function. Biol. Psychiatry 63:990-996.

Davenport, M. D., S. Tiefenbacher, C. K. Lutz, M. A. Novak, and J. S. Meyer. 2006. Analysis of endogenous cortisol concentrations in the hair of rhesus macaques. Gen. Comp. Endocrinol. 147:255-261.

Dobson, H., and R. J. Esslemont. 2002. Stress and its effects on fertility in dairy cows. Adv. Dairy Tech. 14:193-206.

Fisher, D. D., L. L. Wilson, R. M. Leach, and R. W. Scholz. 1985. Switch hair as an indicator of magnesium and copper status of beef cows. Am. J. Vet. Res. 46:2235-2240.

Friend, T. H., F. C. Gwazdauskas, and C. E. Polan. 1979. Change in adrenal response from free stall competition. J. Dairy Sci. 62:768-771.

Friend, T. H., C. E. Polan, F. C. Gwazdauskas, and C. W. Heald. 1977. Adrenal glucocorticoid response to exogenous adrenocorticotropin mediated by density and social disruption in lactating cows. J. Dairy Sci. 60:1958-1963.

González-de-la-Vara, M. R., R. A. Valdez, V. Lemus-Ramirez, J. C. Vazquez-Chagoyan, A. Villa-Godoy, and M. C. Romano. 2011. Ef- fects of adrenocorticotropic hormone challenge and age on hair cortisol concentrations in dairy cattle. Can. J. Vet. Res. 75:216-221.

Huzzey, J. M., D. V. Nydam, R. J. Grant, and T. R. Overton. 2011. Associations of prepartum plasma cortisol, haptoglobin, fecal cortisol metabolites, and nonesterified fatty acids with postpartum health status in Holstein dairy cows. J. Dairy Sci. 94:5878-5889.

Koren, L., O. Mokady, T. Karaskov, J. Klein, G. Koren, and E. Geffen. 2002. A novel method using hair for determining hormonal levels in wildlife. Anim. Behav. 63:403-406.

Lay, D. C., T. H. Friend, R. D. Randel, O. C. Jenkins, D. A. Neuendorff, G. M. Kapp, and D. M. Bushong. 1996. Adrenocorticotropic hormone dose response and some physiological effects of transportation on pregnant Brahman cattle. J. Anim. Sci. 74:1806-1811.

Macbeth, B. J., M. R. L. Cattet, G. B. Stenhouse, M. L. Gibereau, and D. M. Janz. 2010. Hair cortisol concentration as a noninvasive measure of long-term stress in free-ranging grizzly bears (Ursus arctos): Considerations with implications for other wildlife. Can. J. Zool. 88:935-949.

Moberg, G. P., and J. A. Mench, ed. 2000. The Biology of Animal Stress. CAB International, Wallingford, UK.

Moya, D., K. S. Schwartzkopf-Genswein, and D. M. Veira. 2013. Standardization of a non-invasive methodology to measure cortisol in hair of beef cattle. Livest. Sci. 158:138-144.

Möstl, E., J. L. Maggs, G. Schrötter, U. Besenfelder, and R. Palme. 2002. Measurement of cortisol metabolites in faeces of ruminants. Vet. Res. Commun. 26:127-139.

NRC. 2001. Nutrient Requirements for Dairy Cattle. Natl. Acad. Sci. Washington, DC.

Pereg, D., R. Gow, M. Mosseri, M. Lishner, M. Rieder, S. Van Uum, and G. Koren. 2011. Hair cortisol and the risk for acute myocardial infarction in adult men. Stress 14:73-81.

Roth, J. 1985. Cortisol as mediator of stress-associated immunosuppression in cattle. Pages 225-242 in Animal Stress. G. P. Moberg, ed. American Physiological Society, Bethesda, MD.

Roulin, A., B. Almasi, A. Rossi-Pedruzzi, A. L. Ducrest, K. Wakamatsu, I. Miksik, J. D. Blount, S. Jenni-Eiermann, and L. Jenni. 2008. Corticosterone mediates the condition-dependent component of melanin-based coloration. Anim. Behav. 75:1351-1358.

Schwertl, M., K. Auerswald, and H. Schnyder. 2003. Reconstruction of the isotopic history of animal diets by hair segmental analysis. Rapid Commun. Mass Spectrom. 17:1312-1318.

Slominski, A., D. J. Tobin, S. Shibahara, and J. Wortsman. 2004 Melanin pigmentation in mammalian skin and its hormonal regulation. Physiol. Rev. 84:1155-1228.

Stafford, K. J., and D. J. Mellor. 2005. Dehorning and disbudding distress and its alleviation in calves. Vet. J. 169:337-349.

Thomson, S., G. Koren, L. A. Fraser, M. Rieder, T. C. Friedman, and S. H. Van Uum. 2010. Hair analysis provides a historical record of cortisol levels in Cushing's syndrome. Exp. Clin. Endocrinol. Diabetes 118:133-138.

Thun, R., E. Eggenberger, K. Zerobin, T. Lüscher, and W. Vetter 1981. Twenty-four-hour secretory pattern of cortisol in the bull Evidence of episodic secretion and circadian rhythm. Endocrinology 109:2208-2212.

Van Uum, S. H. M., B. Sauve, L. A. Fraser, P. Morley-Foster, T. L. Paul, and G. Koren. 2008. Elevated content of cortisol in hair of patients with severe chronic pain: A novel biomarker for stress. Stress 11:483-488

Yamada, J., B. Stevens, N. de Silva, S. Gibbins, J. Beyene, A. Taddio, C. Newman, and G. Koren. 2007. Hair cortisol as a potential biologic marker of chronic stress in hospitalized neonates. Neonatology 92:42-49. 